Bond University

Research Repository

\title{
Exercise therapy for chronic fatigue syndrome (individual patient data)
}

Larun, Lillebeth; Odgaard-Jensen, Jan; Brurberg, Kjetil G.; Chalder, Trudie; Dybwad,

Marianne; Moss-Morris, Rona E.; Sharpe, Michael; Wallman, Karen; Wearden, Alison; White, Peter D.; Glasziou, Paul P.

Published in:

Cochrane Database of Systematic Reviews

DOI:

10.1002/14651858.CD011040

Licence:

Other

Link to output in Bond University research repository.

Recommended citation(APA):

Larun, L., Odgaard-Jensen, J., Brurberg, K. G., Chalder, T., Dybwad, M., Moss-Morris, R. E., Sharpe, M., Wallman, K., Wearden, A., White, P. D., \& Glasziou, P. P. (2014). Exercise therapy for chronic fatigue syndrome (individual patient data). Cochrane Database of Systematic Reviews, 2014(4), [CD011040].

https://doi.org/10.1002/14651858.CD011040

\section{General rights}

Copyright and moral rights for the publications made accessible in the public portal are retained by the authors and/or other copyright owners and it is a condition of accessing publications that users recognise and abide by the legal requirements associated with these rights.

For more information, or if you believe that this document breaches copyright, please contact the Bond University research repository coordinator. 


\section{(E) Cochrane Library}

Cochrane Database of Systematic Reviews

\section{Exercise therapy for chronic fatigue syndrome (individual patient data) (Protocol)}

Larun L, Odgaard-Jensen J, Brurberg KG, Chalder T, Dybwad M, Moss-Morris RE, Sharpe M, Wallman K, Wearden A, White PD, Glasziou PP

Larun L, Odgaard-Jensen J, Brurberg KG, Chalder T, Dybwad M, Moss-Morris RE, Sharpe M, Wallman K, Wearden A, White PD, Glasziou PP. Exercise therapy for chronic fatigue syndrome (individual patient data).

Cochrane Database of Systematic Reviews 2014, Issue 4. Art. No.: CD011040.

DOI: 10.1002/14651858.CD011040.

www.cochranelibrary.com 


\section{TABLE OF CONTENTS}

HEADER . . . . . . . . . . . . . . . . . . . . . . . . . . . . . . . . . . . . . . . 1

ABSTRACT . . . . . . . . . . . . . . . . . . . . . . . . . . . . . . . . . . . . . . 1

BACKGROUND . . . . . . . . . . . . . . . . . . . . . . . . . . . . . . . . . . 1

OBJECTIVES . . . . . . . . . . . . . . . . . . . . . . . . . . . . . . . . . . . . . .

METHODS . . . . . . . . . . . . . . . . . . . . . . . . . . . . . . . . . . . . . . 4

ACKNOWLEDGEMENTS . . . . . . . . . . . . . . . . . . . . . . . . . . . . . . . . . . . . . . . . .

REFERENCES . . . . . . . . . . . . . . . . . . . . . . . . . . . . . . . . . . . . . . . . . 10

APPENDICES . . . . . . . . . . . . . . . . . . . . . . . . . . . . . . . . . . . . . 12

FEEDBACK . . . . . . . . . . . . . . . . . . . . . . . . . . . . . . . . . . . . . . 20

WHAT'S NEW . . . . . . . . . . . . . . . . . . . . . . . . . . . . . . . . . . . . . . 21

HISTORY . . . . . . . . . . . . . . . . . . . . . . . . . . . . . . . . . . . . 21

CONTRIBUTIONS OF AUTHORS . . . . . . . . . . . . . . . . . . . . . . . . . . . . . . . . . . . . . . .

DECLARATIONS OF INTEREST . . . . . . . . . . . . . . . . . . . . . . . . . . . . . . . . . . . . . . . $\quad$. 22

SOURCES OF SUPPORT . . . . . . . . . . . . . . . . . . . . . . . . . . . . . . . . . . . . . . . . . . 


\section{Exercise therapy for chronic fatigue syndrome (individual patient data)}

Lillebeth Larun $^{1}$, Jan Odgaard-Jensen ${ }^{2}$, Kjetil G Brurberg ${ }^{1}$, Trudie Chalder ${ }^{3}$, Marianne Dybwad ${ }^{4}$, Rona E Moss-Morris ${ }^{5}$, Michael Sharpe $^{6}$, Karen Wallman ${ }^{7}$, Alison Wearden ${ }^{8}$, Peter D White ${ }^{9}$, Paul P Glasziou ${ }^{10}$

${ }^{1}$ Division for Health Services, Norwegian Institute of Public Health, Oslo, Norway. ${ }^{2}$ Medicinrådet, København $\varnothing$, Denmark. ${ }^{3}$ Department of Psychological Medicine, Institute of Psychiatry, Psychology \& Neuroscience, King's College London, London, UK. ${ }^{4}$ Sunnaas Rehabilitation Hospital, Nesoddtangen, Norway. ${ }^{5}$ Section of Health Psychology, Institute of Psychiatry, King's College London, London, UK. ${ }^{6}$ Department of Psychiatry, University of Oxford, Oxford, UK. ${ }^{7}$ School of Sport Science, Exercise and Health, The University of Western Australia, Crawley, Australia. ${ }^{8}$ School of Health Sciences, University of Manchester, Manchester, UK. ${ }^{9}$ Centre for Psychiatry, Barts and The London School of Medicine and Dentistry, Queen Mary University of London, London, UK. ${ }^{10}$ Centre for Research in Evidence-Based Practice (CREBP), Bond University, Gold Coast, Australia

Contact address: Lillebeth Larun, Division for Health Services, Norwegian Institute of Public Health, Postboks 4404 Nydalen, Oslo, N-0403, Norway. lillebeth.larun@fhi.no, lblarun@gmail.com.

Editorial group: Cochrane Common Mental Disorders Group.

Publication status and date: Edited (no change to conclusions), comment added to review, published in Issue 5, 2018.

Citation: Larun L, Odgaard-Jensen J, Brurberg KG, Chalder T, Dybwad M, Moss-Morris RE, Sharpe M, Wallman K, Wearden A, White PD, Glasziou PP. Exercise therapy for chronic fatigue syndrome (individual patient data). Cochrane Database of Systematic Reviews 2014, Issue 4. Art. No.: CD011040. DOI: 10.1002/14651858.CD011040.

Copyright (C) 2018 The Cochrane Collaboration. Published by John Wiley \& Sons, Ltd.

\section{A B S T R A C T}

This is a protocol for a Cochrane Review (Intervention). The objectives are as follows:

To assess the effects of exercise therapy, alone and in combination, for people with chronic fatigue syndrome (CFS) compared with treatment as usual control conditions and other interventions (psychological therapies, pharmacological treatment).

\section{B A C K G R O U N D}

\section{Description of the condition}

Chronic fatigue syndrome (CFS) is an illness characterised by persistent, medically-unexplained fatigue, which is severe enough to result in substantial disability. Fatigue is usually accompanied by other symptoms such as musculoskeletal pain, sleep disturbance, headaches, and impaired concentration and short-term memory (Fukuda 1994; Prins 2006; Sharpe 1991). Some authors believe that myalgic encephalomyelitis (ME) is a separate condi- tion (Carruthers 2011), while others believe that ME and CFS are the same (Medical Research Council 2003). However CFS is the term that has been adopted and clearly defined for research purposes and will be used in this review. The diagnosis requires alternative conditions to be excluded. The prevalence of CFS among adults ranges from $0.2 \%$ to $2.6 \%$ (Prins 2006; Ranjith 2005) and this variation might be related to heterogeneity in diagnostic procedures as there have been at least 20 sets of diagnostic criteria published since 1988 (Brurberg 2014; Christely 2012).

The etiology and pathophysiology of CFS are controversial. Models of understanding can be broadly divided into biomedical and 
biopsychosocial models. The biomedical model explains the illness as caused by abnormalities of the immune, central nervous (Nijs 2011) or endocrine systems and/or a persistent infectious agent. The multifactorial biopsychosocial model (Moss-Morris 2012) distinguishes between precipitating and maintaining factors. Precipitating factors may include acute infective illness and/or excessive stress, while the illness is maintained by the interaction of behavior, thoughts, emotions and physiology. For example, after a severe infection or other illness, attempts to get back to normal life may result in bursts of activity punctuated by the need to rest up to recover, known as all-or-nothing behaviour (Moss-Morris 2010; Spence 2005). These periodic bursts of activity may exacerbate symptoms and result in failure, which further reinforces sufferers' belief that they have a serious, ongoing illness. As time goes by, efforts to meet previous standards of achievement are abandoned and patients become increasingly inactive and distressed by their ongoing symptoms. Inactivity in turn leads to physiological changes such as cardiovascular and muscular deconditioning, dysregulation of the hypothalamic-pituitary-adrenal axis and disrupted circadian rhythms. In this deconditioned state, any activity is liable to produce symptoms, the experience of which reinforces the fearful beliefs and hence reinforces the avoidance of activity (fear avoidance).

\section{Description of the intervention}

There are no curative treatments for CFS and the primary goals of treatment are symptom, function and quality of life management (NICE 2007). Treatment strategies typically involve exercise therapy and cognitive behavioural therapy (CBT) in addition to symptom-specific treatments, such as activity pacing and sleep hygiene. Pharmacotherapy is not an indicated treatment for CFS other than for symptom management or comorbid conditions such as depression or sleep disruption (BMJ Best Practice 2013).

Exercise is physical activity that requires effort (OED 2012). Exercise therapy uses physical exercise to improve health and wellbeing in people who are unwell, and can include modalities such as strength training or endurance exercise given as a part of a goal-oriented, multidisciplinary approach (Rietberg 2004; Takken 2008). There are a number of different approaches to exercise therapy for CFS. These include aerobic exercise such as walking, jogging, swimming or cycling, as well as anaerobic exercise such as strength or stabilising exercises. It is important to look at all kinds of therapeutic exercise, not only aerobic exercise. Behavioral approaches which use carefully graded exercise as a form of systematic desensitisation focus on breaking the fear avoidance cycle and the tendency to interpret symptoms as signs of illness. These approaches usually set a low initial exercise level, which gives patients the opportunity to experience exercise which does not produce a large increase in symptoms. Exercise is then built up gradually in a programme agreed in advance with the patient. If patients experience an increase in symptoms, then planned increases in exercise frequency, duration or intensity are temporarily deferred until symptoms are less intense. Exercise therapy based on improving fitness typically involves setting targets based on physiological indicators of baseline fitness (such as $\mathrm{VO}_{2} \max$ ) and prescribing increases in exercise based on ongoing monitoring of measures of fitness. Whereas both of the above types of exercise therapy include gradual increases in activity over time, a third approach allows patients to reduce activity in response to symptom exacerbations.

Anaerobic exercise, such as pilates, the Mensendieck system and Qigong are stabilising exercises with slow movement, primarily targeting core muscles. Qigong exercise, a method taken from traditional Chinese medicine, is characterised by a low degree of intensity and slow movements, coordinated with breathing and a health-promoting meditative practice. Qigong has been suggested to improve the postural, stabilising muscular system which often gradually progresses to more complex movements (Tsang 2003). Strength training aims to build the strength, anaerobic endurance, and size of skeletal muscles by resistance training, weight training or isometric training, but is also an integral part of other exercise regimes. Strength training exercise is primarily anaerobic.

\section{How the intervention might work}

Various models exist to explain why exercise therapy might be a viable treatment for CFS. The deconditioning model suggests that CFS is perpetuated by a chronic reduction and avoidance of activity leading to reversible physiological changes of deconditioning ( Clark 2005; White 2011). In support of the model, CFS patients show a decreased exercise capacity when compared to sedentary controls (Fulcher 2000), but to date, improvements in fitness following exercise therapy have not been linked to improvements in fatigue in people with CFS (Fulcher 2000; Moss-Morris 2005).

The biopsychosocial model emphasises the role of patients' cognitions and behaviours in the perpetuation of CFS. One variant of this model suggests exercise therapy reduces the focus on symptoms and avoidance of feared activity through showing that gradual increases in activity do not unduly exacerbate symptoms (Moss-Morris 2005), thereby engendering belief change. Reductions in beliefs the about harmful effects of exercise have also been related to improved outcomes in CFS (Deale 1997). A second variant suggests that graded exposure to the previously-avoided exercise extinguishes the conditioned response to exercise.

Further research is needed to verify different hypothesis, but effective treatments may be discovered without knowledge of the effective pathway or underlying cause.

\section{Why it is important to do this review}

Exercise therapy is often used as treatment for CFS both on its own and as part of a treatment program. The available evidence 
(Edmonds 2004; Larun 2011) strongly suggests that exercise therapy might be effective in the treatment of CFS. However it has been reported in one trial that health-related quality of life does not improve with exercise (Nunez 2011) and argued in a review that rest, pacing and conserving energy are better treatments (Twisk 2009). People with CFS should have the opportunity to make decisions about their treatment informed by robust research evidence. This review will examine the effectiveness of exercise therapy, either as a stand-alone intervention or as one part of a treatment plan. Cochrane reviews address many aspects of treatments for CFS. A review of CBT was published in 2008 (Price 2008), and of traditional Chinese herbal medicine in 2009 (Adams 2009).

A meta-analysis of individual participant data (IPD) may be a more reliable method than using only aggregate data meta-analyses. The use of IPD facilitates standardisation of analyses and reporting of results across studies, and allows direct derivation of outcomes, independent of how these were reported (Riley 2010). IPD also increases the power to detect differential treatment effects between the individual participants, providing additional information on who is most likely to respond.

Analysis based on IPD also enables us to use a wider range of statistical and analytical approaches (Higgins 2011a). In particular, it will allow us to explore more thoroughly the relative importance of the aspects of heterogeneity such as type of exercise, intensity, incremental procedures, treatment provided to participants in the control group, baseline illness, and selection criteria, and ensure that missing data and baseline differences are dealt with in standardised ways. Access to IPD also allows subgroup analyses that were not previously reported.

\section{O B J E C T I VES}

To assess the effects of exercise therapy, alone and in combination, for people with chronic fatigue syndrome (CFS) compared with treatment as usual control conditions and other interventions (psychological therapies, pharmacological treatment).

\section{METHODS}

\section{Criteria for considering studies for this review}

\section{Types of studies}

We will include randomised controlled trials, including clusterrandomised trials and cross-over trials, published or unpublished. While every effort will be made to obtain individual participant data (IPD) for trials which meet the selection criteria, an eligible trial will not be excluded if we cannot obtain access to the raw data.

\section{Types of participants}

We will include male and female participants over the age of 17 , irrespective of culture and setting. As several sets of criteria are currently used to diagnose CFS (Sharpe 1991; Fukuda 1994; Carruthers 2011) we will include trials given that the patients fulfil the following diagnostic criteria for CFS:

- Fatigue or a synonym is a prominent symptom;

- Fatigue is medically unexplained (i.e. other diagnosis known to cause fatigue such as psychiatric disorders and cancer should be excluded);

- Fatigue is sufficiently severe to significantly disable or distress the patient; and

- Fatigue has persisted for at least six months.

We will include trials which include patients with disorders other than CFS as long as more than $90 \%$ of the patients had a primary CFS diagnosis according to the criteria above. Trials in which less than $90 \%$ of participants had a primary diagnosis of CFS will only be included in the analysis of this review if data for CFS are reported separately.

\section{Comorbidities}

Studies involving participants with comorbid physical or common mental disorders are eligible for inclusion, as long as the diagnoses of CFS is not excluded by the comorbid condition.

\section{Types of interventions}

\section{Experimental intervention}

Exercise therapy as monotherapy or as an adjunctive treatment (e.g. exercise combined with pharmacological treatment). We define exercise therapy as aerobic or anaerobic interventions aimed at exercising big muscle groups, for example walking, swimming, jogging, strength or stabilising exercises. Both individual and group treatment modalities are eligible, but interventions should be clearly described and supported by appropriate references. We do not impose restrictions with regard to the duration of each treatment session, number of sessions or time between each session. Trials presenting data from one of the following comparisons are eligible for inclusion:

\section{Comparator interventions}

1. Standard care - to include passive conditions of treatment as usual and waiting list, and active conditions of relaxation/ flexibility, pacing and supportive listening. 
2. Psychological therapies - to include cognitive behavioural therapies, psychodynamic therapies and humanistic/supportive therapies.

3. Pharmacological treatments - to include antidepressants, hypnotics, antiviral drugs and immunotherapy.

A non-active supportive listening is regarded as non-active by the trialists and active by participants, whereas a supportive therapy is regarded as active by both trialists and participants.

\section{Types of outcome measures}

Note: 'Validated' is defined as having undergone psychometric development and having been published in a peer-reviewed journal.

\section{Primary outcomes}

\section{Effectiveness}

1. Fatigue, measured using a validated scale (e.g. Fatigue Scale (FS) (Chalder 1993) or the Fatigue Severity Scale (FSS) (Krupp 1989)). 2. Drop out from treatment.

\section{Secondary outcomes}

3. Physical functioning, measured using a validated scale (e.g. SF36, physical functioning sub scale (Ware 1992)).

\section{Safety}

4.Serious adverse reactions and events, measured using any reporting system (e.g. Serious Adverse Reactions (SAR) (European Union Clinical Trials Directive 2001)).

5. Pain, measured using a validated scale (e.g. Visual Analogue Scale for Pain $\left(\mathrm{VAS}_{\text {pain }}\right.$ ) (Finch 2002)).

6. Mood disorders, measured using a validated scale (e.g. Hospital Anxiety and Depression Scale (Zigmond 1983)).

7. Sleep duration and quality, measured using a validated scale (e.g. Pittsburgh Sleep Quality Index (Buysse 1989)).

8. Self-perceived changes in overall health, measured using a validated scale (e.g. Global Impression Scale (Guy 1976)).

9. Symptom severity, treatment response and efficacy, measured using a validated scale (e.g. Clinical Global Impression - Severity scale (CGI-S) (Guy 1976)).

10. Overall functional status, measured using a validated scale (e.g. Work and Social Adjustment Scale (Mundt 2002)).

11. Objective measures of fitness e.g. $\mathrm{VO}_{2}$ max or $\mathrm{VO}_{2}$ at subjective maximal effort, measured for example by 100 watts on an exercise bicycle or running at six miles per hour.

\section{Timing of outcome assessment}

Data on each outcome will be extracted for short-term (end of treatment), medium-term follow up (three to nine months) and long-term follow up (nine months or more).

\section{Search methods for identification of studies}

\section{Electronic searches}

The Cochrane Collaboration's Depression, Anxiety and Neurosis (CCDAN) Review Group's Trials Search Coordinator (TSC) will search their Group's Specialized Register (CCDANCTR-Studies and CCDANCTR-References). This register is created from routine generic searches of MEDLINE (1950-), EMBASE (1974) and PsycINFO (1967- ). Details of CCDAN's generic search strategies, used to inform the CCDANCTR can be found on the Group's website.

The CCDANCTR-Studies Register will be searched using the following terms:

Diagnosis = ("Chronic Fatigue Syndrome" or fatigue) and Free Text $=$ (exercise or sport* or relaxation or "multi convergent" or "tai chi")

The CCDANCTR-References Register will be searched using a more sensitive list of free-text search terms to identify additional untagged/uncoded references, e.g. fatigue*, myalgic encephalomyelitis*, exercise, physical active* and taiji. Full search strategy listed in Appendix 1.

A complementary search of the following bibliographic databases and international trial registers will also be conducted (see Appendix 2):

- SPORTSDiscus (1985 to present);

- The Cochrane Central Register of Controlled Trials (CENTRAL, all years); and

- WHO International Clinical Trials Portal.

\section{Searching other resources}

We will contact the authors of included studies, and screen reference lists to identify additional published or unpublished data. We will also conduct citation searches using the ISI Science Citation Index on the Web of Science.

\section{Data collection and analysis}

\section{Selection of studies}

Two review authors will screen the titles and abstracts obtained from the searches, independently. Trials that appear to fulfil the selection criteria will be noted and full-text articles retrieved. Two 
review authors will independently assess the full-text articles for adherence to the selection criteria. In the case of disagreement, we will attempt to reach a resolution through discussion. Should this prove unsuccessful, a third review author or staff at the CCDAN editorial base will be consulted.

The trialists of the included trials will be invited to take part in a collaborative group by a letter (Appendix 3) stating the main aims and purpose, importance of contribution, publication policy and confidentiality of data. The trialists who provide data will be offered co-authorship according to the Recommendations for the Conduct, Reporting, Editing, and Publication of Scholary Work in Medical Journals (ICMJE 2014). The local secretariat will be based at the Norwegian Knowledge Centre of the Health Services, and the first author will act as project manager. The CCDAN editorial group will act as advisory board. We will ask the trialists to provide their raw data to enable us to assemble the most complete data set possible, including all randomised participants, using flexible data formats containing pseudo-anonymised patient data. The data will be kept and used in accordance with the Norwegian Data Inspectorate's recommendations, and the parties will sign a contract (Appendix 4) to this end. An eligible trial will not be excluded if we cannot obtain access to the raw data.

We will prepare a flow diagram decipiting the flow of references/ studies through the different phases of the review in accordance with the PRISMA statement (Mother 2009). The flow diagram will map out the number of records identified, included and excluded and reasons for exclusion.

\section{Data extraction and management}

Trialists of included studies will be invited to collaborate in accordance with section 18.2 of the Cochrane Handbook (Stewart 2011). They will be offered authorship and asked to provide IPD for all randomised participants to be used in the review. A list of variables that we will particularly request is attached (Appendix 5), but we will accept data sets containing more variables than requested. We will accept all data sent in data formats that can be read by SAS or SPSS, Microsoft Access databases, Excel and delimited or (comma-)separated text-files. The review authors will adopt data security measures to ensure data protection, and to ensure that the data cannot be violated or tracked. All data sets will be stored securely and pseudo-anonymously; that is, all identifiers that potentially could be linked directly to the actual participants will be deleted, and identifiers will only be identifiable to the original investigators.

Once the raw data is received from the trialists, checks for consistency and comparison with results presented in the journal papers, will be performed by JO-J. Any queries arising from these checks will be resolved in cooperation with the trialists. All analyses based on the Individual Patient Data from the included trials for this review will be undertaken by JO-J, while meta-analyses will be performed by JO-J and KGB, independent of trialists. Descriptive data (methodology, treatment, comparator, and instruments used for measuring outcomes) for each of the included studies will be independently extracted by JO-J and LL using a standardised data collection form (Appendix 6) which will include the following variables.

Study methodology specific:

- Diagnostic criteria used for identifying eligible patients;

- Method of recruitment for trial;

- Randomisation method;

- Allocation concealment; and

- Blinding.

Treatment specific:

- Deliverer of intervention;

- Explanation and material;

- Type of exercise;

- Schedule, therapist;

- Schedule, home;

- Duration of sessions;

- Initial exercise level;

- Increment steps;

- Patient self monitoring; and

- Criteria for (non) increment.

Outcome specific:

Scales used for assessment

- Fatigue;

- Pain;

- Physical functioning;

- Depression;

- Anxiety; and

- Quality of life.

Measurement time points/follow-up will be collected and study authors contacted to provide additional information where gaps have been identified.

For included studies from which we are not able to gain access to IPD, relevant information (both the above-mentioned data and results for the prespecified outcomes) will be extracted. For adverse events, we will extract the number of events and number of participants in each group. For the remaining (continuous) outcomes, we will extract $\mathrm{N}$, mean and standard deviation.

\section{Planned comparisons}

1. Exercise therapy versus treatment as usual/waiting list/ supportive listening/pacing/cognitive treatment. We also plan to conduct subgroup comparisons for each comparison above to see if one of the more passive comparators is more effective than another.

2. Exercise therapy (as monotherapy or adjunctive therapy) versus cognitive behavioural therapy. Monotherapy and adjunctive therapy will be analysed separately. 
3. Exercise therapy (as monotherapy or adjunctive therapy) versus pharmacological treatment.

If there are several drug trials we will divide the comparators into antidepressants, hypnotics, antiviral and immunotherapy. Monotherapy and adjunctive therapy will be analysed separately.

\section{Data checking and cleaning}

To reduce potential bias (Tierney 2005), we will request information for all randomised patients including those who had been excluded from the investigators' original analyses. A number of standard checks will be applied to all incoming trials, including checks for missing values, data validity and consistency across variables. To assess the randomisation integrity, we will look for unusual patterns in the sequencing of allocation or imbalances in baseline characteristics between treatment arms. Follow-up of patients will be assessed to ensure that it was balanced in the treatment arm, and as up-to-date as possible.

All incoming data will be checked thoroughly for consistency and completeness of follow-up. We will tabulate summary measures (frequencies for categorical variables and mean, standard deviations, minimum, maximum, median, 25th and 75th percentile for continuous variables) for the individual parameters (demographic variables, illness-specific variables and outcomes) for each study to identify missing data and outliers, and to describe differences in distribution between the studies. We will analyse patient and disease characteristics and treatment outcome by trial and treatment arm to check consistency with published results. If we encounter any problems regarding missing data, obvious errors, discrepancies (e.g. between published data and raw data), inconsistencies between variables or extreme values or inability to replicate the results presented in the retrieved papers, we will resolve these in discussion with the original investigator. We will maintain a log of all changes made to the data originally supplied by the trialists, and the reasons for these changes. Any queries will be resolved and the final database entries verified by the responsible trial investigator or statistician.

As the summary statistics for outcomes in this review may differ from the summary statistics presented in the retrieved papers due to the possible use of imputed data in the original analyses by trial investigators, we will present summary statistics for all outcomes by trial and treatment arm in a table (showing sample size, mean, standard deviation, minimum and maximum).

\section{Assessment of risk of bias in included studies}

Two authors (LL and JO-J) will use the Cochrane Collaboration's tool for assessing risk of bias (Higgins 2011a), published in the most recent version of the Cochrane Handbook (Higgins 2011b). This tool encourages consideration of how the allocation sequence was generated, how allocation was concealed, the integrity of blinding at outcome level, the completeness of outcome data, selective outcome reporting (only applicable in cases where we do not gain access to complete data sets) and other potential sources of bias. When it comes to blinding, we will distinguish between performance bias (blinding of participants and personnel) and detection bias (blinding of outcome assessors; for outcomes not reported/assessed by the participants). As all the outcomes we intend to look at are subjective (self report by participants, or the use of scales that are based on judgements) we will include an item for objective outcomes. Each item in the 'Risk of bias' assessment will be assessed as low, high or unclear risk of bias using the guidelines outlined in the Cochrane Handbook (Higgins 2011a). In addition, we will make an overall assessment of the risk of bias across all items for each included study. If one or more of the items sequence generation, allocation concealment or completeness of outcome data are assessed as being at high risk of bias, the overall assessment of the study will be high risk of bias.

We will perform sensitivity analyses in which studies assessed to be at high risk of bias (across all items) are excluded. The reasons for the judgement 'high risk of bias' might vary between studies where IPD are available and studies where IPD are not available. Selective outcome reporting will only be a problem for non IPDstudies as we have access to all data from the included studies with IPD. Furthermore we can reduce the risk of bias due to non-completeness of outcome data for studies with IPD by using statistical methods that do not exclude participants based on missing data (such as analysis of longitudinal data, or the use of censoring when analysing time-to-event data).

\section{Measures of treatment effect}

\section{Binary data}

We will calculate the odds ratio (OR) and its 95\% confidence interval (CI).

\section{Continuous data}

When the same scale has been used in all included studies, we will calculate the mean differences (MD) and their 95\% CI, as it preserves the original units and is therefore easier to interpret. Where different scales are used for the same outcome, effect sizes will be calculated separately for each scale.

\section{I Change versus endpoint data}

Mean differences will primarily be based on endpoint data. For studies where IPD are not available, we will only use change data when endpoint data are not available. In cases where results from some studies are based on change data and results from other studies are based on endpoint data, all studies will be included in the same meta-analysis with change data and endpoint data as subgroups. 


\section{Unit of analysis issues}

\section{Cluster-randomised trials}

Studies increasingly employ 'cluster randomisation' (such as randomisation by clinician or practice) but analysis and pooling of clustered data poses problems. Firstly, authors often fail to account for intra-class correlation in clustered studies, leading to a 'unit of analysis' error (Divine 1992) whereby P values are spuriously low, confidence intervals unduly narrow and statistical significance overestimated. This causes type I errors (Bland 1997; Gulliford 1999).

Where clustering is not accounted for in primary studies, we will present data in a table, with a $\left(^{*}\right)$ symbol to indicate the presence of a probable unit of analysis error. We will seek to contact first authors of studies to obtain the intra-class correlation co-efficient (ICC) of their clustered data and to adjust for this using accepted methods (Gulliford 1999). Where clustering is incorporated, we will present the data as if from a parallel-group randomised study, but adjusted for the clustering effect. We will additionally exclude such studies using a sensitivity analysis.

If cluster studies are appropriately analysed taking into account ICC and relevant data documented in the report, synthesis with other studies will be possible using the generic inverse variance technique.

\section{Cross-over trials}

A major concern of cross-over trials is the potential for carryover effect. It occurs if an effect (e.g. pharmacological, physiological or psychological) of the treatment in the first phase is carried over to the second phase. As a consequence on entry to the second phase the participants can differ systematically from their initial state despite a wash-out phase. For the same reason cross-over trials are not appropriate if the condition of interest is unstable (Elbourne 2002). As both effects are very likely in CFS/ME, randomised cross-over studies will be eligible for inclusion, but only data up to the point of first cross-over will be used, while data from the following (second) period of the cross-over trial will not be considered for analysis. This might introduce bias due to the possibility of selectively reporting results from the first period based on the results. We will exclude cross-over studies using a sensitivity analysis.

\section{Studies with multiple treatment groups}

\section{Multiple dose groups}

We expect that some studies will address the effects of different levels of supervision and follow-up in regards to the exercise intervention to the comparator (e.g. sessions for designing exercise therapy, sessions for designing exercise therapy and planned telephone contacts, sessions for designing exercise therapy and seven face to face treatment sessions, and usual care). In the case of dichotomous outcomes we will sum up the sample sizes and the number of people with events across all intervention groups. For continuous outcomes, we will combine means and standard deviations using methods described in Chapter 7 (section 7.7.3.8) of the Cochrane Handbook (Higgins 2011b).

\section{Multiple medications}

We expect that some other studies will combine several interventions with one comparison group. In this case we will analyse the effects of each intervention group versus placebo separately, but will divide up the total number of participants in the placebo group. In the case of continuous outcomes the total number of participants in the placebo group will again be divided up, but the means and standard deviations will be left unchanged (see chapter 16, section 16.5.4 in Higgins 2011b).

\section{Dealing with missing data}

Analyses of all endpoints, subsets and subgroups will carried out on the basis of the intention-to-treat principle but based on the available data; that is, participants will be analysed according to their allocated treatment, irrespective of whether they received that treatment or not, but no attempt will be made to impute missing data. In our request for the raw data from the included trials it will be made clear that data are needed for all randomised participants.

\section{Assessment of heterogeneity}

We will assess clinical heterogeneity across the included trials in terms of interventions, participants and settings. We expect that the trials might differ when it comes to the ingredients of the active interventions and the components of the passive controls (treatment as usual, waiting list, etc.). We furthermore anticipate that the severity and duration of CFS might differ between the trials. If we judge that the included trials are too heterogeneous (e.g. we expect the effect sizes across trials to be unrelated) to warrant a formal meta-analysis, we will not perform meta-analysis but present the results of the included trials narratively.

We will assess statistical heterogeneity on the basis of the Cochrane Handbook recommendations ( $\mathrm{I}^{2}$ values of $0 \%$ to $40 \%$ : might not be important; $30 \%$ to $60 \%$ : may represent moderate heterogeneity; $50 \%$ to $90 \%$ : may represent substantial heterogeneity; $75 \%$ to $100 \%$ : considerable heterogeneity). In addition to the $\mathrm{I}^{2}$ value (Higgins 2003), we will present the $\chi^{2}$ and its P value and consider the direction and magnitude of the treatment effects. As in meta-analysis with few studies, the $\chi^{2}$ test is underpowered to detect heterogeneity should it exist; a P value of 0.10 is used as a threshold of statistical significance. 


\section{Assessment of reporting biases}

Reporting biases arise when the dissemination of research findings is influenced by the nature and direction of results. These biases included publication bias and selective outcome reporting bias. Funnel plots can be useful in reporting biases (Sterne 2011, section 10.4). It is important to bear in mind when interpreting a funnel plot, that publication and selective outcome reporting biases are not the only reasons for asymmetry. Poor methodological quality of studies, inappropriate analysis and true heterogeneity between trials can also lead to funnel plot asymmetry.

We will only produce funnel plots if at least 10 studies are included for that specific outcome and the studies are not similar in size. Funnel plots will be inspected visually and Egger's test for asymmetry (Egger 1997) used to assess the risk of reporting bias. We will interpret the results with caution; all test results will be assessed in light of the visual inspection. We acknowledge that tests for funnel plot symmetry in general have relatively low power to detect funnel plot asymmetry. Thus bias cannot be excluded even if the test does not provide evidence of funnel plot asymmetry. For studies where we obtain IPD, we do not consider selective outcome reporting to be a problem, as we have access to all data collected. For the remaining non-IPD studies, selective outcome reporting can be an issue, which will be addressed during the 'Risk of bias' assessment.

\section{Data synthesis}

Data from the included studies will be analysed using a two-step approach (Riley 2008).

At the first step, we will analyse the IPD for each trial, separately. For continuous outcomes the study-level analyses will be based on repeated measurements with a reference group coding of independent factors, thus taking into account the correlation between baseline and post-intervention measurements. Data from all measurement points will be included in one single model. The post intervention measurements will be modelled as depending on the baseline measurement, time. group (intervention or control) and the interaction between time and group. The repeated measurements (from the same person) will be assumed to have an unstructured covariance structure. The analyses of data from the individual included trials will be conducted using the MIXED procedure in SAS (SAS 2009). For each trial the estimate of effect at any given measurement point will be calculated as the difference between the estimated value of the dependent variable in the intervention and control groups, respectively (at that measurement point); the corresponding $95 \%$ confidence intervals will also be calculated.

If a study has performed more than one measurement of the same outcome at baseline, the baseline value for our analysis will be defined as the last value. If measurement of an outcome has been performed more than once at each measurement point, then we will base our analysis on the first-measured value.
For studies where no IPD are available, the estimate of effect (MD with standard error) will be based on the sample sizes, means, standard deviations, confidence intervals and $\mathrm{P}$ values extracted from papers.

At the second step we will combine the estimates of effect across studies in meta-analysis. The primary analyses will be based on all included studies, both IPD and non-IPD, but we will conduct sensitivity analyses in which studies where IPD are not available will be excluded. The estimates of effect from all included studies will be pooled using the generic inverse variance technique in a random-effects model.

Adverse events will be counted and reported for each study using odds ratios (ORs) and their 95\% confidence intervals. A randomeffects model will be used to estimate effects across studies.

We will use GRADE to examine the quality of evidence and the strength of recommendations. Judgment of the strength of a recommendation will require consideration of the following factors: the balance between benefit and harm, the quality of the evidence, translation of the evidence into specific circumstances and the certainty of the baseline risk (Guyatt 2008).

\section{Subgroup analysis and investigation of heterogeneity}

To examine the potential impact of trial design and the treatments used, we plan to group trials by important aspects that might influence the effect of exercise therapy.

Heterogeneity will be explored on the following trial-specific items:

- Control groups (treatment as usual/waiting list versus relaxation/flexibility);

- Diagnostic criteria used for assessing eligibility of participants (Oxford (Sharpe 1991) versus CDC1994 (Fukuda 1994) versus London ME criteria (The London Criteria 1994) versus ICC (Carruthers 2011)); and

- Setting (primary versus secondary versus tertiary care).

For each of these analyses a pooled measure of treatment effects will be calculated for each group of trials and for all trials together. Participant-specific items:

- Age (less than 45 years of age versus 45 years or older);

- Gender;

- Length of syndrome history (less then 5 years versus more than 5 years);

- Baseline illness severity (dichotomised);

- Baseline anxiety (Yes/No);

- Baseline depression (Yes/No);

- Comorbidity (Yes/No);

- Diagnostic criteria met (Met least strict criteria versus met two sets of criteria versus met three sets of criteria versus met all four sets of criteria); and

- Illness beliefs (virus/psychological/combination).

We will furthermore explore heterogeneity on the following intervention-specific items: 
- Baselining (no determination/patient-centred/ physiological);

- Type of exercise (aerobic/anaerobic-strengthening/ anaerobic-non-strengthening);

- Explanations and materials (no cognitive component/ educational-didactic/educational-didactic plus therapist using cognitive approaches); and

- Incremental steps (none/pacing/mutually planned and expected/physiological response to exercise).

For the participant-specific items we will calculate separate estimates of treatment effect for each subgroup using the same methods as for the main analyses. Pooled measures of treatment effect will be calculated for each subgroup of the population, but not across subgroups due to the dependency between estimates of effect from the same trial.

If enough trials are included (a minimum of 10 studies with all relevant data available per comparison), meta-regression (randomeffects) will be performed to formally explore heterogeneity or differences between subgroups of trials or populations. We will perform one meta-regression for each variable we wish to explore (not adjusted for the other proposed subgroup variables). The meta-regression will be conducted using the MIXED procedure with random error terms for each trial in SAS (SAS 2009). Due to the number of subgroup analyses we will adjust the level of significance to $0.05 /$ (\#number of subgroup analyses) using the Bonferroni correction method.

\section{Sensitivity analysis}

Sensitivity analysis will be performed, separately, by excluding:

- studies assessed as being at high risk of bias on one or more of the 'Risk of bias' items: sequence generation, allocation concealment or completeness of outcome data;

- cluster-randomised trials;

- cross-over trials (possible selective reporting of results from first period in non-IPD studies); and

- studies where IPD are not available (estimates of treatment effect depends on the method of analysis).

We will, in addition, perform sensitivity analyses based on standardised mean differences (SMD) for continuous outcomes that have been measured using different instrument/scales in the included studies. For the outcome Clinical Global Impression Scale (CGI) we will perform sensitivity analyses based on dichotomised values:
- Participant became better ( 1 and 2 versus 3 to 7); and

- Participant became worse (6 and 7 versus 1 to 5).

\section{'Summary of findings' tables}

We will prepare 'Summary of findings' tables to summarise the key findings of the systematic review in line with the standard methods described in the Cochrane Handbook for Systematic Reviews of Interventions (Higgins 2011b) using GRADEpro (Brozek 2008). These findings will include:

- Fatigue;

- Drop out rates;

- Physical functioning; and

- Self-perceived changes in overall health.

\section{ACKNOWLEDGEMENTS}

We would like to thank Jane Dennis and Hugh MacGuire for getting us started.

The author team held three meetings in 2011, 2012 and 2013 which were funded as follows:

2011 via Paul Glasziou, NIHR senior research fellow fund, Oxford Department of primary care.

2012 via Hege R Eriksen, Uni Research Health, Bergen.

2013 via Peter D White's academic fund (Professor of Psychological Medicine, Centre for Psychiatry, Wolfson Institute of Preventive Medicine, Barts and The London School of Medicine and Dentistry, Queen Mary University of London).

\section{CRG Funding Acknowledgement}

The National Institute for Health Research (NIHR) is the largest single funder of the Cochrane Depression, Anxiety and Neurosis Group.

\section{Disclaimer}

The views and opinions expressed herein are those of the authors and do not necessarily reflect those of the NIHR, NHS or the Department of Health. 


\section{REFERE N C E S}

\section{Additional references}

\section{Adams 2009}

Adams D, Wu T, Yang X, Tai S, Vohra S. Traditional Chinese medicinal herbs for the treatment of idiopathic chronic fatigue and chronic fatigue syndrome. Cochrane Database of Systematic Reviews 2009, Issue 4. DOI: 10.1002/14651858.CD006348.pub2

Bland 1997

Bland JM, Kerry SM. Statistics notes. Trials randomised in clusters. BMJ 1997;315:600.

BMJ Best Practice 2013

BMJ Best Practice. Chronic fatigue syndrome Step-by step. http://bestpractice.bmj.com/best-practice/monograph/277/ treatment/step-by-step.html 2013.

\section{Brozek 2008 [Computer program]}

Brozek J, Oxman A, Schünemann H. GRADEpro. Version 3.2 for Windows. Brozek J, Oxman A, Schünemann $\mathrm{H}$, 2008.

Brurberg 2014

Brurberg KJ, Fonhus MS, Larun L, Flottorp S, Malterud $\mathrm{K}$. Case definitions for chronic fatigue syndrome/ myalgic encephalomyelitis (CFS/ME): a systematic review. BMJ Open 2014;4(2):e003973. DOI: 10.1136/ bmjopen-2013-003973

Buysse 1989

Buysse DJ, Reynolds CF, Monk TH, Berman SR, Kupfer DJ. The Pittsburgh Sleep Quality Index: a new instrument for psychiatric practice and research. Psyciatric Research 989; 1(28): 193-213.

\section{Carruthers 2011}

Carruthers BM, an de Sande MI, Meirleir KL, Klimas NG, Broderick G, Mitchell T, et al. Myalgic encephalomyelitis: International Consensus Criteria. Journal of Internal Medicine 2011;270(4):327-38. DOI: 10.1111/ j.1365-2796.2011.02428.x

\section{Chalder 1993}

Chalder T, Berelowitz G, Pawlikowska T, Watts L, Wessely $\mathrm{S}$, Wright D, et al. Development of a fatigue scale. Journal of Psychosomatic Research 1993;37(6):147-53.

\section{Christely 2012}

Christely Y, Duffy T, Martin CR. A review of the definitional criteria for chronic fatigue syndrome. Clinical Practice 2012; 18:25-31. DOI: 10.1111/j.1365-2753.2010.01512.x

\section{Deale 1997}

Deale A, Chalder T, Marks I, Wessely, S. Cognitive behavior therapy for chronic fatigue syndrome: a randomized controlled trial. American Journal of Psychiatry 1997;154(3): $408-14$.

Divine 1992

Divine GW, Brown JT, Frazer LM. The unit of analysis error in studies about physicians' patient care behavior. Journal of General Internal Medicine 1992;7:623-9.
Edmonds 2004

Edmonds M, McGuire H, Price J. Exercise therapy for chronic fatigue syndrome. Cochrane Database of Systematic Reviews 2004, Issue 3. DOI: 10.1002/ 14651858.CD003200.pub2

\section{Egger 1997}

Egger M, Smith GD, Schneider M, Minder C. Bias in meta-analysis detected by a simple, graphical test. $B M J$ 1997:315:629-34.

\section{Elbourne 2002}

Elbourne DR, Altman DG, Higgins JP, Curtin F, Worthington HV, Vail A. Meta-analyses involving crossover trials: methodological issues. International Journal of Epidemiology 2002;31:140-9.

European Union Clinical Trials Directive 2001

The European Parliament and the Council of the European Union. DIRECTIVE 2001/20/EC European Parliament and the Council of the European Union of 4 April 2001. Official Journal of the European Communities 2001;L 121/ 34. http://www.eortc.be/services/doc/clinical-eu-directive04-april-01.pdf.

Finch 2002

Finch E, Brooks D, Stratford PW, Mayo N. Physical Rehabilitation Outcome Measures. A Guide to Clinical Decision Making. 2nd Edition. Canadian Physiotherapy Association, Canada: Lippincott, Williams and Wilkins, 2002.

\section{Fukuda 1994}

Fukuda K, Straus SE, Hickie I, Sharpe MC, Dobbins JG, Komaroff A. The chronic fatigue syndrome; a comprehensive approach to its definition and study. Annals of Internal Medicine 1994;121(12):953-9.

\section{Fulcher 2000}

Fulcher KY, White PD. Strength and physiological response to exercise in patients with chronic fatigue syndrome. Journal of Neurology, Neurosurgery and Psychiatry 2000;69: $302-7$.

\section{Gulliford 1999}

Gulliford MC, Ukoumunne OC, Chinn S. Components of variance and intra class correlations for the design of community-based surveys and intervention studies: data from the Health Survey for England 1994. American Journal of Epidemiology 1999;149:876-83.

\section{Guy 1976}

Guy WR. ECDEU Assessment Manual for Psychopharmacology. Rockville, MD: National Institute of Mental Health, 1976

\section{Guyatt 2008}

Guyatt GH, Oxman AD, Vist GE, Kunz R, Falck-Ytter Y, Alonso-Coello P, et al. GRADE: an emerging consensus on rating quality of evidence and strength of recommendations. BMJ 2008;336(7650):924-6. 


\section{Higgins 2003}

Higgins JPT, Thompson SG, Deeks JJ, Altman DG. Measuring inconsistency in meta-analyses. BMJ 2003;327 (7414):557-60.

\section{Higgins 2011a}

Higgins JPT, Altman DG, Sterne JAC (editors). Chapter 8: Assessing risk of bias in included studies. In: Higgins JPT, Green S editor(s). Cochrane Handbook for Systematic Reviews of Interventions Version 5.1.0 [updated March 2011] Available from www.cochrane-handbook.org. The Cochrane Collaboration, 2011.

Higgins 2011b

Higgins JPT, Green S (editors). Cochrane Handbook for Systematic Reviews of Interventions Version 5.1.0 [updated March 2011]. The Cochrane Collaboration. Available from www.cochrane-handbook.org, 2011.

ICMJE 2014

International Committee of Medical Journal Editors (ICMJE). Recommendations for the Conduct, Reporting, Editing and Publication of Scholarly Work in Medical Journals (ICMJE Recommendations). http:// www.icmje.org/recommendations/ 2011-2013.

\section{Krupp 1989}

Krupp LB, LaRocca NG, Muir-Nash J, Steinberg AD. The Fatigue Severity Scale. Application to patients with multiple sclerosis and systemic lupus erythematosus. Archives of Neurology 1989;46:1121-3.

\section{Larun 2011}

Larun L, Malterud K. Finding the right balance of physical activity. A focus group study about experiences among patients with chronic fatigue syndrome. Patient Education and Counseling 2011;83:222-6.

Medical Research Council 2003

Medical Research Council. MRC CFS/ME Research Advisory Group, CFS/ME Research Strategy. NHS, Medical Research Council 2003.

\section{Moss-Morris 2005}

Moss-Morris R, Sharon C, Tobin R, Baldi JC. A randomized controlled graded exercise trial for chronic fatigue syndrome: outcomes and mechanisms of change. Journal of Health Psychology 2005;10(2):245-59.

\section{Moss-Morris 2010}

Moss-Morris R, Spence MJ, Hou R. The pathway form glandular fever to chronic fatigue syndrome: can the cognitive behavioural model provide the map?. Psychological Medicine 2010;00:1-9.

Moss-Morris 2012

Moss-Morris R, Deary V, Castell B. Chronic fatigue syndrome. In: Barnes M, Good D editor(s). NeuroRehabilitation in the Handbook of Clinical Neurology 3rd Series. Elsevier, 2012:303-14.

Mother 2009

Moher D, Liberati A, Tetzlaff J, Altman DG. Preferred Reporting Items for Systematic Reviews and Meta-Analyses: The PRISMA Statement. PLoS Medicine 2009;6(7): e1000097. DOI: 10.1371/journal.pmed1000097

\section{Mundt 2002}

Mundt JC, Marks IM, Shear MK, Greist JM. The Work and Social Adjustment Scale: a simple measure of impairment in functioning. The British Journal of Psychiatry 2011;180: 461-4. DOI: 10.1192/bjp.180.5.461

\section{NICE 2007}

National Collaborating Center for Primary Care. NICE clinical guideline 53. Chronic fatigue syndrome/myalgic encephalomyelitis (or encephalopathy): diagnosis and management of CFS/ME in adults and children. National Institute for Health and Clinical Excellence 2007.

\section{Nijs 2011}

Nijs J, Meeus M, Van Oosterwijck J, Ickmans K, Moorkens G, Hans G, et al. In the mind or the brain? Scientific evidence for central sensitisation in chronic fatigue syndrome. European Journal of Clinical Investigation 2011; 42:203-11. DOI: 10.1111/j.1365-2362.2011.02575.x]

\section{Nunez 2011}

Núñez M, Fernández-Solà J, Nuñez E, Fernández-Huerta J-M, Godás-Sieso T, Gomez-Gil E. Health-related quality of life in patients with chronic fatigue syndrome: group cognitive behavioural therapy and graded exercise versus usual treatment. A randomised controlled trial with 1 year of follow-up. Clinical Rheumatology 2011;30:381-9. DOI: 10.1007/s10067-010-1677-y

OED 2012

Oxford Dictionaries. The Oxford English Dictionary. Oxford University Press, 2012. [978-0-19-861424-1]

Price 2008

Price JR, Mitchell E, Tidy E, Hunot V. Cognitive behaviour therapy for chronic fatigue syndrome in adults. Cochrane Database of Systematic Reviews 2008, Issue 3. DOI: 10.1002/14651858.CD001027.pub2

\section{Prins 2006}

Prins JB, van den Meer JW, Bleijenberg G. Chronic fatigue syndrome. Lancet 2006;367:346-55.

Ranjith 2005

Ranjith G. Epidemiology of chronic fatigue syndrome. Occupational Medicine 2005;55(1):13-9. DOI: 10.1093/ occmed/kqi012

\section{Rietberg 2004}

Rietberg MB, Brooks D, Uitdehaag BMJ, Kwakkel G. Exercise therapy for multiple sclerosis. Cochrane Database of Systematic Reviews 2004, Issue 3. DOI: 10.1002/ 14651858.CD003980

\section{Riley 2008}

Riley RD, Lambert PC, Staessen JA, Wang J, Gueyffier F, Thijs L, et al. Meta-analysis of continuous outcomes combining individual patient data and aggregate data. Statistics in Medicine 2008;27(11):1870-93. DOI: $10.1002 /$ sim. 3165

\section{Riley 2010}

Riley RD, Lambert PC, Abo-Zaid G. Meta-analysis of individual participant data: rationale, conduct, and reporting. BMJ 2010;340:521-5. 


\section{SAS 2009 [Computer program]}

SAS Institute Inc. SAS. Version 9.2. Cary, NC: SAS Institute Inc, 2009.

\section{Sharpe 1991}

Sharpe M, Archard L, Banatvala J, Borysiewicz LK, Clare AW, David A, et al. Chronic fatigue syndrome: guidelines for research. Journal of the Royal Society of Medicine 1991;84 (2):118-21.

Spence 2005

Spence M, Moss-Morris R, Chalder T. The Behavioural Responses to Illness Questionnaire (BRIQ): a new predictive measure of medically unexplained symptoms following acute infection. Psychological Medicine 2005;35(4):583-93.

Sterne 2011

Sterne JAC, Egger M, Moher D (editors). Chapter 10: addressing reporting biases. In: Higgins JPT, Green S editor(s). Cochrane Handbook for Systematic Reviews of Interventions Version 5.1.0 [updated March 2011] Available from www.cochrane-handbook.org. The Cochrane Collaboration, 2011.

Stewart 2011

Stewart LA, Tierney JF, Clarke M. Chapter 18: Reviews of individual patient data. In: Higgins JPT, Green S editor(s). Cochrane Handbook of Systematic Reviews, version 5.1.0. The Cochrane Collaboration, 2011.

Takken 2008

Takken T, Van Brussel M, Engelbert RH, van der Net JJ, Kuis W, Helders PPJM. Exercise therapy in juvenile idiopathic arthritis. Cochrane Database of Systematic Reviews 2008, Issue 2. DOI: 10.1002/14651858.CD005954.pub2

The London Criteria 1994

The London criteria. Report on chronic fatigue syndrome (CFS), post viral fatigue syndrome (PVFS) and myalgic encephalomyelitis (ME). Westcare, Bristol: The National Task Force 1994.

\section{Tierney 2005}

Tierney JF, Stewart LA. Investigating patient exclusion bias in meta-analysis. International Journal of Epidemiology 2005;34(1):79-87.

Tsang 2003

Tsang WW, Hui-Chan CW. Effects of tai chi on joint proprioception and stability limits in elderly subjects. Medicine and Science in Sports and Exercise 2003;35(12): 1962-71.

Twisk 2009

Twisk FNM, Maes M. A review on cognitive behavorial therapy (CBT) and graded exercise therapy (GET) in myalgicencephalomyelitis (ME)/chronic fatigue syndrome (CFS). Neuroendocrinology Letters 2009;30(3):284-99.

\section{Ware 1992}

Ware JE, Sherbourne CD. The MOS 36-item short form health survey (SF-36). Medical Care 1992;30:473-83.

\section{Zigmond 1983}

Zigmond AS, Snaith RP. The Hospital Anxiety and Depression Scale. Acta Psychiatrica Scandinavica 1983;67 (6):361-70

* Indicates the major publication for the study

\section{A P P E N D I C ES}

\section{Appendix I. Search strategy - CCDANCTR-References}

\section{The Cochrane, Depression, Anxiety and Neurosis Review Group's Specialised Register (CCDANCTR)}

The Cochrane Depression, Anxiety and Neurosis Group (CCDAN) maintain two clinical trials registers at their editorial base in Bristol, UK, a references register and a studies based register. The CCDANCTR-References Register contains over 34,000 reports of randomized controlled trials in depression, anxiety and neurosis. Approximately $60 \%$ of these references have been tagged to individual, coded trials. The coded trials are held in the CCDANCTR-Studies Register and records are linked between the two registers through the use of unique Study ID tags. Coding of trials is based on the EU-Psi coding manual. Please contact the CCDAN Trials Search Coordinator for further details. Reports of trials for inclusion in the Group's registers are collated from routine (weekly), generic searches of MEDLINE (1950-), EMBASE (1974-) and PsycINFO (1967-); quarterly searches of the Cochrane Central Register of Controlled Trials (CENTRAL) and review specific searches of additional databases. Reports of trials are also sourced from international trials registers c/o the World Health Organisation's trials portal ( ICTRP), ClinicalTrials.gov, drug companies, the hand-searching of key journals, conference proceedings and other (non-Cochrane) systematic reviews and meta-analyses.

Details of CCDAN's generic search strategies can be found on the Group's website.

There will be no restriction on date, language or publication status applied to the search.

CCDANCTR-Studies and CCDANCTR-References Register will be searched using the following terms: \#1. "chronic fatigue"

Exercise therapy for chronic fatigue syndrome (individual patient data) (Protocol) 


\section{\#2. CFS:ti,ab}

\#3. ((fatigue* or asthenia or "muscular disorder*" or neurasthenia* or "infectious mononucleos" or "myalgic encephalomyelit*" or "royal free disease*" or lassitude or "muscular weakness*" or "akureyri disease" or "atypical poliomyelitis" or CFIDS or CFS or (chronic and mononucleos*) or "epidemic neuromyasthenia" or "iceland disease" or "post infectious encephalomyelitis" or PVFS or tiredness or adynamia or legasthenia or (perspective and asthenia) or neurataxia or ("muscle strength" and loss) or "muscle* weak" or "weak* muscle*" or (muscular and insufficiency) or (neuromuscular and fatigue)) AND (sport* or exercise or "physical fitness" or "physical education" or "physical condition*" or "physical train*” or "physical mobility" or "physical activ*" or "physical exertion" or "physical effort" or "physical medicine" or (breathing and (therap* or exercise*)) or (respiration and therap*) or "qi gong" or qigong or qitherapy or *kung or tai or thai or taiji or taijiquan or taichi or walking or hiking or yoga or relaxation* or gymnastics or calisthenics or aerobic or danc* or jumping or hopping or running or jogging or ambulat* or "muscle strengthening" or (muscular and (strength or resistance)) or ((weight or weights) and lifting) or weightlifting or "power lifting" or "weight train*" or pilates or stretching or plyometric* or "cardiopulmonary conditioning" or "motion therap*" or "neuromuscular facilitation"” or "movement therap*" or ((recreation or activity) and therap*) or "isometric training" or climbing or cycling or bicycl* or "lifting effort" or swim* or aquatic or (training and (technical or course or program*)) or writing or kinesi* or gardening or multiconvergent))

\#4. (\#1 or \#2 or \#3)

$\# 5$. cancer

\#6. (\#4 not \#5)

\section{Appendix 2. Other search strategies}

\section{Cochrane Central Register of Controlled Trials (CENTRAL)}

\#1 MeSH descriptor: [Exercise] explode all trees

\#2 MeSH descriptor: [Exercise Therapy] explode all trees

\#3 MeSH descriptor: [Exercise Movement Techniques] explode all trees

\#4 MeSH descriptor: [Physical Fitness] this term only

\#5 MeSH descriptor: [Physical Education and Training] explode all trees

\#6 MeSH descriptor: [Sports] explode all trees

\#7 exercis*:ab,kw,ti

\#8 ((breathing or respiration) near/2 (therap* or exercis*)):ab,kw,ti

\#9 relaxation:ab,kw,ti

\#10 ("qi gong" or qigong or gigong or "chi gung" or "chi gong" or "chi kung"):ab,kw,ti

\#11 yoga:ab,kw,ti

\#12 ("tai chi“" or tai-chi* or taiji or taijiquan or taichi or tai-yi or tai-ji):ab,kw,ti

\#13 (physical near/2 (fitness or condition* or education or training or mobility or activit* or exertion or effort)):ab,kw,ti

\#14 (gymnastics or calistenics):ab,kw,ti

$\# 15$ aerobic*ab,kw,ti

\#16 danc*:ab,kw,ti

\#17 (jumping or hopping):ab,kw,ti

\#18 (walking or jogging or running):ab,kw,ti

\#19 ambulat*:ab,kw,ti

\#20 (muscle next strengthening):ab,kw,ti

\#21 (muscular near/2 (strength or resistance)):ab,kw,ti

\#22 ((weight or weights) near/2 lift*):ab,kw,ti

\#23 (weightlifting or power next lifting or weight next training):ab,kw,ti

\#24 (Pilates or stretching or plyometric* or cardiopulmonary next conditioning or motion next therap* or neuromuscular next fa? cilitation* or movement next therap* or gymnastic next therap* or isometric next training or climbing or cycling or lifting next effort* or swimming or writing):ab, kw, ti

\#25 ((recreation or activity) near/2 therap*):ab,kw,ti

\#26 "technical training ":ab,kw,ti

\#27 (training near/2 (course* or program*)):ab,kw,ti

\#28 kinesi?therap*:ab,kw,ti

\#29 gardening:ab,kw,ti

Exercise therapy for chronic fatigue syndrome (individual patient data) (Protocol)

Copyright ( 2018 The Cochrane Collaboration. Published by John Wiley \& Sons, Ltd. 
\#30 multiconvergent:ab,kw,ti

$\# 31$ (\#1 or \#2 or \#3 or \#4 or \#5 or \#6 or \#7 or \#8 or \#9 or \#10 or \#11 or \#12 or \#13 or \#14 or \#15 or \#16 or \#17 or \#19 or \#21 or \# 22 or \#23 or \#24 or \#25 or \#26 or \#27 or \#28 or \#29 or \#30)

\#32 MeSH descriptor: [Fatigue Syndrome, Chronic] this term only

\#33 MeSH descriptor: [Asthenia] this term only

\#34 MeSH descriptor: [Neurasthenia] this term only

\#35 (chronic next fatigue*):ab,kw,ti

\#36 (fatigue next syndrom*):ab,kw,ti

\#37 (infectious next mononucleos*):ab,kw,ti

\#38 (chronic next fatigue-fibromyalgia next syndrome*):ab,kw,ti

\#39 (myalgic next encephalomyelit*):ab,kw,ti

\#40 (royal next free next disease*):ab,kw,ti

\#41 (neurast?enic next neuros*):ab,kw,ti

\#42 ((akureyri or iceland*) next disease):ab,kw,ti

\#43 "atypical poliomyelitis“:ab,kw,ti

\#44 (CFIDS or CFS):ab,kw,ti

\#45 (chronic near/5 mononucleos*):ab,kw,ti

\#46 "epidemic neuromyasthenia“: $a b, k w, t i$

\#47 (post next infectious next encephalomyelit*):ab,kw,ti

\#48 PVFS:ab,kw,ti

\#49 (perspective near/5 ast?enia):ab,kw,ti

\#50 (neurast?enic next syndrome*):ab,kw,ti

\#51 neurataxia:ab,kw,ti

\#52 neurast?enia:ab,kw,ti

\#53 (neuromus?ular near/6 fatigue):ab,kw,ti

$\# 54$ (\#32 or \#33 or \#34 or \#35 or \#36 or \#37 or \#38 or \#39 or \#40 or \#41 or \#42 or \#43 or \#44 or \#45 or \#46 or \#47 or \#48 or \#49

or \#50 or \#51 or \#52 or \#53)

\#55 \#31 and \#54 [in Trials]

SPORTSDiscus (EBSCOHost)

S67 (S38 AND S61 AND S66)

S66 (S62 OR S63 OR S64 OR S65)

S65 TI (((singl* or doubl* or tripl*) W0 (blind* or mask* or dummy)) ) OR AB ( ((singl* or doubl* or tripl*) W0 (blind* or mask* or dummy)))

S64 TI ((control* or prospectiv* or volunteer*)) OR AB ((control* or prospectiv* or volunteer*))

S63 TI ((clinic* N2 (trial? or study or studies))) OR AB ((clinic* N2 (trial? or study or studies)))

S62 TI ((randomi?ed or randomly or placebo? or trial?)) OR AB ( (randomi?ed or randomly or placebo? or trial?))

S61 (S39 OR S41 OR S42 OR S43 OR S44 OR S45 OR S46 OR S47 OR S48 OR S49 OR S50 OR S51 OR S52 OR S53 OR S54

OR S55 OR S56 OR S57 OR S58 OR S59 OR S60)

S60 TI (neuromuscular N5 fatigue) OR AB (neuromuscular N5 fatigue)

S59 TI neuroasthenia OR AB neuroasthenia

S58 TI neurataxia OR AB neurataxia

S57 TI (neurasthenic W0 syndrome*) OR AB (neurasthenic W0 syndrome*)

S56 TI (perspective N3 asthenia) OR AB (perspective N3 asthenia)

S55 TI PVFS OR AB PVFS

S54 TI post-infectious W0 encephalomyelitis OR AB post-infectious W0 encephalomyelitis

S53 TI epidemic W0 neuromyasthenia OR AB epidemic W0 neuromyasthenia

S52 TI (chronic N4 mononucleos*) OR AB (chronic N4 mononucleos*)

S51 TI ((CFIDS or CFS) ) OR AB ( (CFIDS or CFS))

S50 TI atypical W0 poliomyelitis OR AB atypical W0 poliomyelitis

S49 TI ((akureyri or iceland) W0 disease) OR AB ((akureyri or iceland) W0 disease)

S48 TI neurasthenic W0 neuros* OR AB neurasthenic W0 neuros*

S47 TI royal W0 free W0 disease* OR AB royal W0 free W0 disease*

S46 TI myalgic W0 encephalomyelit* OR AB myalgic W0 encephalomyelit*

Exercise therapy for chronic fatigue syndrome (individual patient data) (Protocol)

Copyright ( 2018 The Cochrane Collaboration. Published by John Wiley \& Sons, Ltd. 
S45 TI chronic W0 fatigue-fibromyalgia W0 syndrome* OR AB chronic W0 fatigue-fibromyalgia W0 syndrome*

S44 TI infectious W0 mononucleos* OR AB infectious W0 mononucleos*

S43 TI fatigue W0 syndrom* OR AB fatigue W0 syndrom*

S42 TI chronic W0 fatigue* OR AB chronic W0 fatigue*

S41 SU Asthenia OR SU Neurasthenia

S39 SU Fatigue Syndrome, Chronic

S38 (S1 OR S2 OR S3 OR S4 OR S5 OR S6 OR S7 OR S8 OR S9 OR S10 OR S11 OR S12 OR S13 OR S14 OR S15 OR S16 OR S17 OR S 18 OR S19 OR S20 OR S21 OR S22 OR S23 OR S24 OR S25 OR S26 OR S27 OR S28 OR S29 OR S30 OR S31 OR S32 OR S33 OR S34 OR S35 OR S36 OR S37)

S37 TI multiconvergent OR AB multiconvergent

S36 TI gardening OR AB gardening

S35 TI kinesi?therap* OR AB kinesi?therap*

S34 TI ((training W0 (course* or program*)) ) OR AB ( (training W0 (course* or program*)))

S33 TI technical W0 training OR AB technical W0 training

S32 TI writing OR AB writing

S31 TI lifting W0 effort* OR AB lifting W0 effort*

S30 TI (climbing or cycling or swimming) OR AB (climbing or cycling or swimming)

$\mathrm{S} 29 \mathrm{TI}$ isometric W0 training $\mathrm{OR} \mathrm{AB}$ isometric W0 training

S28 TI gymnastic W0 therap* OR AB gymnastic W0 therap*

S27 TI (((recreation or activity) W0 therap*)) OR AB (((recreation or activity) W0 therap*))

S26 TI neuromuscular W0 fa?cilitation* OR AB neuromuscular W0 fa?cilitation*

S25 TI (((motion or movement) W0 therap*)) OR AB (((motion or movement) W0 therap*))

S24 TI cardiopulmonary W0 conditioning OR AB cardiopulmonary W0 conditioning

S23 TI plyometric* OR AB plyometric*

S22 TI stretching OR AB stretching

S21 TI pilates OR AB pilates

S20 TI (((weight? N1 lifting) or weightlifting or power W0 lifting or weight W0 training) ) OR AB ( ((weight? N1 lifting) or weightlifting or power W0 lifting or weight W0 training))

S19 TI (((muscular or muscle) W0 (strength or resistance) W0 training)) OR AB (((muscular or muscle) W0 (strength or resistance) W0 training))

S18 TI muscle W0 strengthening OR AB muscle W0 strengthening

S17 TI ambulat* OR AB ambulat*

S16 TI ((jumping or hopping or jogging or running) ) OR AB ( (jumping or hopping or jogging or running))

S15 TI (aerobic W0 danc* or danc*) OR AB (aerobic W0 danc* or danc*)

S14 TI (gymnastics or calisthenics) OR AB (gymnastics or calisthenics)

S13 TI ((physical W0 (fitness or condition* or education or training or mobility or activit* or exertion or effort))) OR AB ( (physical W0 (fitness or condition* or education or training or mobility or activit* or exertion or effort)))

S12 TI yoga OR AB yoga

S11 TI walking OR AB walking

S10 TI ("tai-chi*“ or taiji or taijiquan or taichi or "tai-yi“" or "tai-ji“) OR AB ("tai-chi*“ or taiji or taijiquan or taichi or "tai-yi“" or "taiji“)

S9 TI ("qi gong" or qigong or gigong or "chi gung" or "chi gong" or "chi kung") OR AB ("qi gong" or qigong or gigong or "chi gung" or "chi gong" or "chi kung")

S8 TI relaxation* OR AB relaxation*

S7 TI (((breathing or respiration) W0 (therap* or exercise*)) ) OR AB ( ((breathing or respiration) W0 (therap* or exercise*)))

S6 TI ((exercise* or exercising)) OR AB ((exercise* or exercising))

S5 SU "physical education \& training“+

S4 SU physical fitness

S3 SU "exercise therapy“+

S2 SU exercise+

S1 SU sports+

International Trial Registers

Exercise therapy for chronic fatigue syndrome (individual patient data) (Protocol)

Copyright (c) 2018 The Cochrane Collaboration. Published by John Wiley \& Sons, Ltd. 
WHO International Clinical Trials Portal available at: http://apps.who.int/trialsearch/, incorporating the following International trials registers/registries:

- Australian New Zealand Clinical Trials Registry

- ClinicalTrials.gov

- EU Clinical Trials Register (EU-CTR)

- ISRCTN

- Brazilian Clinical Trials Registry (ReBec)

- Chinese Clinical Trial Registry

- Clinical Trials Registry - India

- Clinical Research Information Service - Republic of Korea

- Cuban Public Registry of Clinical Trials

- German Clinical Trials Register

- Iranian Registry of Clinical Trials

- Japan Primary Registries Network

- Pan African Clinical Trial Registry

- Sri Lanka Clinical Trials Registry

- The Netherlands National Trial Register

- Thai Clinical Trials Register (TCTR)

\section{Appendix 3. Letter of invitation}

Dear Dr XXX,

Following up our previous communication on the need to follow up and elaborate on the the Cochrane review 'Chronic Fatigue Syndrome and graded exercise therapy' from 2004; your study, XXXX, is one of the studies to be included.

As previously mentioned we intend to conduct an Individual Participant Data (IPD) review. The reason for the IPD review is to enable in-depth analyses by performing subgroup analyses. This will be carried out using variables such as patient, illness and treatment characteristics. We are able to handle data delivered in the following formats:

- Excel

- SAS

- SPSS/PASW Statistics

- Delimited or fixed-width files provided that a detailed file-description with variable-names, formats and maximum width of each column is provided.

Please don't hesitate to contact us if you need more information. Enclosed is a memorandum which should be returned to us with the datafiles.

We would like to invite you to participate as co-author(s) in line with your contribution. Should you not wish to be listed as a coauthor you will of course be mentioned under Acknowledgments for the provision of your raw data.

The review will be undertaken in collaboration with representatives from Uni Health Bergen and The Norwegian Knowledge Centre for the Health Services, Oslo, as well as the authors of the included studies.

Yours sincerely

Lillebeth Larun

Attachments

- Memorandum to be signed

- List of background variables

- Data extraction template

Exercise therapy for chronic fatigue syndrome (individual patient data) (Protocol) 


\section{Appendix 4. Memorandum}

\section{Exercise therapy for chronic fatigue syndrome (individual patient data)}

Whereas:

The parties have agreed to provide information which they consider to be confidential in nature (the "Confidential Information") to write the review: 'Exercise Therapy for Chronic Fatigue Syndrome (Individual Patient Data)', hereafter called the "Purpose”;

\section{It is agreed as follows:}

1. In consideration of each of the parties disclosing to the other Confidential Information for the Purpose the parties hereby undertake that they shall:

i) not communicate, disclose or make available all or any of the part of the Confidential Information to any third party

ii) not directly or indirectly use, or permit others to use, the Confidential Information other than for the Purpose;

iii) not make any announcements or disclosure in connection with the Confidential Information or the Purpose without prior consent of the other party.

2. The obligation of the confidentiality and non-use will not apply to any of the following:

i) information which is generally available to the public at the date of this agreement;

ii) information which is subsequently disclosed by third parties having no obligation of confidentiality;

iii) information which is or will become generally available to the public in printed publications in general circulation through no act or default on the parties.

3. Without prejudice to the generality of clause 2 information shall not be deemed to be generally available to the public by reason only that it is known to only a few of those people to whom it may be of commercial interest and a combination of two or more parts of the Confidential Information shall not be deemed to be generally available to the public by reason only of separate part being so available.

4. The parties shall each ensure that all measures necessary are taken to secure the confidentiality of the other party's Confidential Information including but not limited to:

i) keeping separate all Confidential Information and all information generated based on the Confidential Information from all other documents and records;

ii) keeping all documents and any other material bearing or incorporating any of the Confidential Information at the party's usual place of business;

iii) not using, reproducing, transforming or storing any of the $\mathrm{V}$ in an external accessible computer or electronic information retrieval system, not transmitting it in any form or by any means outside the party's usual place of business and not copying all or any part of the Confidential Information without the prior consent and then only to the extent that the same is required for the Purpose;

iv) allowing access to the Confidential Information only to those employees and/or to the professional advisers who have reasonable need to see or use it for the Purpose and informing each of the said employees and professional advisers of the confidential nature of the Confidential Information and of the obligations in respect of the Confidential Information and ensuring such employees and professional advisers comply with the confidentiality and non-disclosure obligations in this agreement;

v) obtaining form employees having access to the Confidential Information their undertakings to maintain the same as confidential and taking such steps as may be reasonable to enforce such obligations;

vi) delivering all documents and other materials in the possessions, custody or control of the party, employees or professional advisers that bear or incorporate any part of the Confidential Information of the other party;

vii) delivering all data material encrypted wherever possible and make sure that individual participants cannot be identified form the data provided.

5. The failure by any party to enforce at any time any one or more of the terms or conditions of this agreement shall not be a waiver of them or of the right at any time subsequently to enforce all terms and conditions in this agreement.

6. The parties agree that damages might not be sufficient remedy to any breach of the terms of this agreement and that as a result injunctive or other equitable relief may be obtained in respect of any breach or anticipated breach.

7. All right in the Confidential Information are reserved by the party to which it belongs and no rights or obligations other than those expressly set out in this agreement are granted or to be implied form this agreement. In particular no license is granted directly or indirectly by this agreement relating to any intervention, discovery, patent, copyright or other industrial or intellectual property right now or in future held, made, obtained or licensable by either party.

Exercise therapy for chronic fatigue syndrome (individual patient data) (Protocol)

Copyright (c) 2018 The Cochrane Collaboration. Published by John Wiley \& Sons, Ltd. 
8. The rights, duties and obligations of the parties and the validity, interpretation, performance and legal effect of this agreement shall be governed and determined by the laws of country of residence.

AGEED by the parties:

SIGNED by

Name (print)

Position

Date (Day/Month/Year)

SIGNED by

Name (print)

Position

Date (Day/Month/Year)

\section{Appendix 5. List of variables requested from original investigators}

\section{Gender (M/F)}

Age (years)

\section{Education}

Martial status

Income

Employment status

Complied with protocol $(\mathrm{Y} / \mathrm{N})$

Completed follow-up (Y/N)

Number of sessions attended

Duration of illness (years)

Illness severity

Previous diagnosis (specify)

Co-morbidity (specify)

previous treatment

Adjunctive treatment

Illness perception

Medication 
(Continued)

Physical fatigue

Mental fatigue (all measurements)

Total fatigue (all measurements)

Percieved exertion (all measurements)

Depression (all measurements)

Anxiety (all measurements)

Pain (all measurements)

Functional impairment (all measurements)

Quality of life (all measurements)

Adverse outcomes (specify)

\section{Appendix 6. Data collection form - descriptive data}

\section{Study specific}

Diagnostic criteria for identify-

ing eligible patients

Methods of recruitment for trial

Randomisation method

Allocation concealment

Blinding (who was blinded to

what)

\section{Treatment specific}

Deliverer of intervention

Explanation and material

Type of exercise

Exercise therapy for chronic fatigue syndrome (individual patient data) (Protocol) 
(Continued)

Schedule, therapist

Schedule, home

Duration of sessions

Initial exercise level

Increment steps

Patient self monitoring

Criteria for (non) increment

Scales used for assessment Name Self-reported or clinician-reported (please When measured state by whom and whether the clinician was

blinded to treatment allocation)

Fatigue

Pain

Functional impairment

Depression

Anxiety

Quality of life

FEE D B A C K

Exercise therapy for chronic fatigue syndrome (individual patient data), 20 March 2018

\section{Summary}

In October 2016 a FOI request was submitted to Queen Mary University London QMUL for additional data from the PACE trial which was an included study in the previous Cochrane Review on Exercise for CFS. ( http://www.cochrane.org/CD003200/ DEPRESSN_exercise-treatment-patients-chronic-fatigue-syndrome). The PI of the PACE trial is now a co-author on this IPD review. The freedom of information request was rejected in November 2016 citing an exemption in the law that covers data which is part of ongoing research or scheduled to be included in future publications. Does this refer to the future publication of this review and the previous review update?

An internal appeal to QMUL was rejected, and the complainant appealed to the Information Commissioners Office (ICO) in March 2017. In the interim, the PI of the PACE trial retired from QMUL.

Exercise therapy for chronic fatigue syndrome (individual patient data) (Protocol)

Copyright (C) 2018 The Cochrane Collaboration. Published by John Wiley \& Sons, Ltd. 
During this time, QMUL changed its rationale for not providing the data saying that they were not available because the PI had gone and they are not required to hire staff to access data in response to FOI requests.

The PI of the PACE Trial is both an author of the Cochrane review and the PI of the PACE trial. So he should be able to provide all the data needed for the new review. However, QMUL told the ICO that they are the holder and owner of the raw data and have lost the means to locate and extract it because that requires specialist knowledge. I would argue that the patients that gave their time and bodies to participate in the trial, and the people who paid taxes to fund the research are the owners of the data, and to deny access to them is unethical.

Does this mean the Cochrane reviewers will also be unable to access all the raw data from the PACE trial despite the PI being on the author team?

Submitter name: Caroline Struthers

Role: Patient advocate

\section{Reply}

Your comment includes two specific questions in relation to availability of data from the PACE trial.

Your first question relates to the correspondence between QMUL and ICO, in which QMUL has cited an exemption in the law, which covers data in ongoing research or future publications. The authors of the Cochrane review have, however, not been involved in the correspondence between QMUL and ICO, and we have no knowledge of what QMUL has referred to in their response.

Your second question relates to the data availability for the Cochrane reviewers in relation to the data from the PACE trial. We can confirm that the Cochrane reviewers will have access to sufficient PACE trial data to perform the review in accordance with the Cochrane protocol (with it's appendices).

In addition, your comment contains certain statements relating to the FOI request submitted to QMUL, and their responses on the current availability of raw data from the PACE trial. The Cochrane reviewers have no knowledge of these matters, and can neither confirm nor reject the statements made in your comment. If someone submits a request for data related to this Cochrane review, it should however be noted, that the reviewers have a duty of confidentiality on terms equal to those outlined in Appendix 4 in the protocol.

\section{Contributors}

Response from Lillebeth Larun (first author)

\section{WHAT'S NEW}

\begin{tabular}{lll}
\hline Date & Event & Description \\
\hline 10 May 2018 & Feedback has been incorporated & Feedback comment and response added. \\
\hline
\end{tabular}

\section{H I S T O R Y}

Protocol first published: Issue 4, 2014 


\begin{tabular}{|c|c|c|}
\hline Date & Event & Description \\
\hline 20 May 2014 & Amended & $\begin{array}{l}\text { Following questions around the involvement of trialists in the analysis, the protocol has been updated } \\
\text { to clarify how independence will be ensured. Minor amendments were also made to the search strategy }\end{array}$ \\
\hline
\end{tabular}

\section{CONTRIBUTIONS OFAUTHORS}

Lillebeth Larun (LL), Jan Odgaard-Jensen (JO-J) and Kjetil Gundro Brurberg (KGB) developed and drafted the protocol.

Hugh McGuire and Sarah Dawson, CCDAN Trials Search Coordinators, developed the search with input from LL.

JO-J developed and drafted the statistical analysis.

All the co-authors have contributed to the final draft of the protocol.

\section{DECLARATIONS OF INTEREST}

All analyses for this review will be undertaken by JO-J and KGB independently of trialists providing data.

KGB: none known.

TC: may contribute anonymised individual patient data from one or more trials, and has received royalties from Sheldon Press and Constable and Robinson for self help books related to chronic fatigue.

MD: none known; may contribute anonymised individual patient data from one or more trials.

PG is on the board of Therapeutic Guidelines Limited, and holds a research grant from the NHMRC on screening and diagnostic testing.

LL: none known.

REM-M: may contribute anonymised individual patient data from one or more trials, and is a national adviser to NHS England for Increasing Access to Psychological Therapies for People with Long Term and Medically Unexplained Conditions.

JO-J: none known.

MS: may contribute anonymised individual patient data from one or more trials, and has done occasional consultancy for Aegon Insurance Ltd.

KW: none known; may contribute anonymised individual patient data from one or more trials.

AW: none known; may contribute anonymised individual patient data from one or more trials.

PDW: may contribute anonymised individual patient data from one or more trials, and has undertaken both voluntary and paid consultancy work for the UK government and a re-insurance company 


\section{SOURCES OF SUPPORT}

\section{Internal sources}

- Norwegian Knowledge Centre for the Health Services, Norway. Wages for JO-J, KGB and LL

\section{External sources}

- No sources of support supplied 